- Original Article

\title{
Higher Serum Calcium Levels Are Associated with Preclinical Peripheral Arterial Disease among the Apparently Healthy Individuals
}

\author{
Hyung-Jin Kim, Mi-Ri Kim, Jin-Kyung Park, Yong-Jae Lee, Byoungjin Park* \\ Department of Family Medicine, Yonsei University College of Medicine, Seoul, Korea
}

Background: Epidemiological studies suggest that serum calcium levels correlate with cardiovascular events. An ankle-brachial index (ABI) between 0.9 and 1.00 is a surrogate estimation of preclinical peripheral arterial disease (PAD). Prior studies have shown that an ABI of 0.9-1.0 is also associated with endothelial dysfunction. Therefore, we sought to investigate the relationship between serum calcium levels and preclinical PAD in apparently healthy Korean individuals.

Methods: We evaluated the association between serum calcium levels and preclinical PAD in 596 participants (334 males, 262 females) in a health examination program. Preclinical PAD was defined by an ABI of 0.9-1.0. Multiple logistic regression analysis was used to determine whether the serum calcium level was an independent determinant of preclinical PAD.

Results: The overall prevalence of preclinical PAD was $14.3 \%$. The mean age was $44.0 \pm 12.5$ years in the non-PAD group and $48.3 \pm 11.4$ years in the preclinical $\mathrm{PAD}$ group $(\mathrm{P}=0.001)$. After adjusting for age, gender, systolic blood pressure, fasting plasma glucose, high-density lipoprotein cholesterol, triglycerides, C-reactive protein, $\gamma$-glutamyltransferase, uric acid, hypertension medication, diabetes medication, and hyperlipidemia medication, the odds ratio (95\% confidence intervals) for preclinical PAD was $2.28(1.02-5.11)$ with a $1-\mathrm{mg} / \mathrm{dL}$ increase in the serum calcium.

Conclusion: These findings suggest that increased serum calcium is independently and positively associated with preclinical PAD regardless of the presence of classic cardiovascular risk factors.

Keywords: Serum Calcium Levels; Preclinical Peripheral Arterial Disease; Ankle Brachial Index; Cardiovascular Diseases

Received: March 6, 2017, Revised: June 15, 2017, Accepted: July 11, 2017

*Corresponding Author: Byoungjin Park https://orcid.org/0000-0003-1733-5301

Tel: +82-31-331-8710, Fax: +82-31-331-5551, E-mail: bjpark96@yuhs.ac 


\section{INTRODUCTION}

Calcium is a pervasive divalent cation that is involved in blood coagulation, skeletal mineralization, muscle withdrawal, and nerve sensitivity. It is finely managed by the balance of calcitonin and parathyroid hormone (PTH). ${ }^{1,2)}$ Epidemiological data over time suggest that serum calcium levels are associated with cardiovascular events. ${ }^{1)}$ Calcium supplementation is thought to be beneficial for bone health in children, young adults, the elderly, and menopausal females. However, there is also a concern regarding the possible relationship between serum calcium and incident cardiovascular illness. ${ }^{1)}$ Elevated serum calcium may have an early effect on cardiovascular morbidity through its effects on arterial stiffness. ${ }^{3)}$ Several mechanisms may explain calcium's effects on cardiovascular morbidity. An increased serum calcium level affects pyrophosphate, which is an important inhibitor of tissue calcification, by binding to the calcium-sensing receptor and altering endothelial function. In addition, elevated serum calcium levels can influence blood coagulation, thereby increasing the rates of cardiovascular events and mortality. ${ }^{3)}$

The ankle-brachial index (ABI) is a simple, noninvasive clinical test that is not only useful in the diagnosis of peripheral arterial disease (PAD) but also in yielding important prognostic information about future cardiovascular events. ${ }^{4-9)}$ Measuring the ABI involves recording the systolic pressures in the brachial artery at each elbow, in the posterior tibial artery, and in the dorsalis pedis arteries at each ankle. ${ }^{4,9,10)}$ The ratio of the ankle systolic pressure to the higher brachial pressure can then be ascertained. ${ }^{4,10-12)}$ The ABI is calculated for each leg separately, and the lower of the two values is considered the result. ${ }^{9,12} \mathrm{PAD}$ is diagnosed by an $\mathrm{ABI}$ of $\leq 0.9$ and graded as mild to moderate if the $\mathrm{ABI}$ is between 0.4 and 0.9 and severe if $<0.400^{5-7,10)} \mathrm{An} \mathrm{ABI}$ value $>1.4$ is also considered abnormal and suggestive of non-compressible vessels. ${ }^{5-7,10)}$

Previous studies have found ABIs of 0.9-1.00 to be associated with endothelial dysfunction. ${ }^{5)}$ The association between serum calcium level and borderline ABI (as a surrogate marker of preclinical PAD) is poorly understood. Therefore, we sought to investigate the relationship between serum calcium level and preclinical PAD, as defined by an ABI of 0.9-1.0, in apparently healthy Korean individuals.

\section{METHODS}

\section{Study Population}

We reviewed the medical records of 728 individuals (416 men, 312 women) who voluntarily underwent a medical examination at a health promotion center in the tertiary university hospital between March 2006 and May 2007. Individuals were excluded if they met any of the following criteria $(\mathrm{n}=45)$ : missing covariate information, $\mathrm{ABI}<0.9$ or $>1.4$, or C-reactive protein >3.0. Ultimately, 596 participants (334 males, 262 females) were included in the final analysis. This study was approved by the Institutional Review Board of the medical institutions (IRB approval no., 3-2017-0201). Informed consent was obtained from each participant.

\section{Data Collection}

The medical staff performed every examination according to the same procedures. Participants were asked about their lifestyle (including cigarette smoking, alcohol consumption), physical activity (more or less than two times per week), and whether or not they were currently being treated for any disease. If the patients were currently being treated for a specific condition, they were asked for the date of diagnosis and a list of their current medications. Trained staff reviewed the completed questionnaires and entered the responses into a database. Participants were also categorized into non-drinkers, abstainers $(<140 \mathrm{~g} /$ wk of alcohol), or current drinkers ( $\geq 140 \mathrm{~g} /$ wk of alcohol). Body weight and height were measured in light indoor clothing (without shoes) to the nearest $0.1 \mathrm{~kg}$ and $0.1 \mathrm{~cm}$, respectively. Body mass index (BMI) was calculated as the weight divided by the height squared $\left(\mathrm{kg} / \mathrm{m}^{2}\right)$.

Blood samples were drawn from an antecubital vein after a 12-hour overnight fast. Fasting plasma glucose, high-density lipoprotein (HDL) cholesterol, triglycerides, C-reactive protein, $\gamma$-glutamyltransferase (GGT), and uric acid were measured using a Hitachi 7600-110 Chemistry system Autoanalyzer (Hitachi, Tokyo, Japan). Blood pressure was measured using an automated device (TM-2665; A\&D Co. Ltd., Tokyo, Japan) with the participant in a seated position after five minutes of rest. Diabetes was defined as a self-reported history or by a fasting plasma glucose level $\geq 126 \mathrm{mg} / \mathrm{dL}$. Hypertension was defined as a selfreported history, systolic blood pressure $\geq 140 \mathrm{~mm} \mathrm{Hg}$, or diastolic blood pressure $\geq 90 \mathrm{~mm} \mathrm{Hg}$.

\section{Ankle-Brachial Index Acquisition}

An automated oscillometric device (Form PWV/ABI; BP-203PRE II Instrument Omron Colin Co., Komaki, Japan) was used to calculate ABI by simultaneously measuring the systolic pressures at bilateral brachial and ankle arteries. With the subject in the supine position, systolic blood pressures were measured at the brachial arteries and in both the dorsalis pedis and posterior tibial arteries of the lower extremities. ${ }^{4,10,12)}$ The ABI was calculated separately for each leg by dividing the higher of the two ankle systolic pressures by the higher of the two brachial systolic pressures. ${ }^{4,10)}$ According to the definition from the American Heart Association scientific statement, the ABI is the ratio of the systolic blood pressure measured at the ankle to that at the brachial artery. $^{10,12)}$

\section{Statistical Analyses}

The demographic and biochemical characteristics of the study population according to preclinical PAD were compared using independent two-sample t-tests for continuous variables and the chi-square test for categorical variables. A multiple logistic regression analysis was performed to determine whether the serum calcium level is an independent determinant of preclinical PAD. All analyses were conducted using the SAS statistical software ver. 9.1 (SAS Institute Inc., Cary, NC, USA). All statistical tests were two-sided. Statistical significance was 
determined by a P-value $<0.05$.

\section{RESULTS}

Table 1 shows the general characteristics of the study population $(\mathrm{n}=596)$. The overall prevalence of preclinical PAD was $14.3 \%$. The mean age was $44.0 \pm 12.5$ years in the non-PAD group and $48.3 \pm 11.4$ years in the preclinical PAD group ( $\mathrm{P}=0.001)$. The mean $\mathrm{BMI}\left(\mathrm{kg} / \mathrm{m}^{2}\right)$ was 23.8 \pm 2.9 in the control group and 23.5 \pm 2.9 in the preclinical PAD group $(\mathrm{P}=0.404)$. Fasting plasma glucose and $\mathrm{C}$-reactive protein levels were higher in the preclinical PAD group than in the control group, although these differences were not statistically significant.

Figure 1 shows the unadjusted spline curve between serum calcium levels and preclinical PAD. The probability of preclinical PAD gradually increased according to serum calcium. Figure 2 shows the spline curve between serum calcium levels and preclinical peripheral arterial disease after adjusting for the same variables on Table 2, and the results showed that the logarithm of odds ratio for $\mathrm{ABI}$ lower than 1.0 tends to be increasing according to serum calcium level.

Table 2 shows the risk of preclinical PAD according to changes in the serum calcium levels. After adjusting for age, gender, systolic blood pressure, fasting plasma glucose, HDL cholesterol, triglycerides, C-reactive protein, GGT, uric acid, hypertension medication, diabetes medication, and hyperlipidemia medication the odds ratio (95\% CIs) of preclinical PAD was $2.28(1.02-5.11)$ with a $1-\mathrm{mg} / \mathrm{dL}$ increase in the calcium level $(\mathrm{P}=0.046)$. In addition, the odds ratio (95\% confidence intervals) for a history of hyperlipidemia medication for preclinical $\mathrm{PAD}$ was 3.67 (1.33-10.14; $\mathrm{P}=0.012$ ).

\section{DISCUSSION}

In this population based, cross-sectional study, there was a positive association between higher serum calcium levels and preclinical PAD (independent of classic cardiovascular risk factors). Preclinical PAD

Table 1. Characteristics of the study population

\begin{tabular}{|c|c|c|c|c|}
\hline Characteristic & Total $(n=596)$ & Control $(n=511)$ & $\begin{array}{c}\text { Preclinical peripheral arterial } \\
\text { disease }(n=85)\end{array}$ & P-value \\
\hline Age (y) & $47.7 \pm 11.6$ & $44.0 \pm 12.5$ & $48.3 \pm 11.4$ & 0.001 \\
\hline Body mass index $\left(\mathrm{kg} / \mathrm{m}^{2}\right)$ & $23.7 \pm 2.9$ & $23.8 \pm 2.9$ & $23.5 \pm 2.9$ & 0.404 \\
\hline Current smoker & 22.2 & 23.7 & 12.9 & 0.027 \\
\hline Alcohol consumption & 71.5 & 71.6 & 70.6 & 0.845 \\
\hline Regular exercise & 59.1 & 57.1 & 70.6 & 0.020 \\
\hline Systolic blood pressure (mm Hg) & $119.5 \pm 15.3$ & $120.0 \pm 15.7$ & $116.8 \pm 12.6$ & 0.077 \\
\hline Fasting plasma glucose (mg/dL) & $96.1 \pm 18.4$ & $96.0 \pm 15.4$ & $96.7 \pm 15.4$ & 0.840 \\
\hline Triglyceride (mg/dL) & $112.7 \pm 94.0$ & $114.1 \pm 97.1$ & $104.0 \pm 72.2$ & 0.966 \\
\hline High-density lipoprotein cholesterol (mg/dL) & $48.1 \pm 12.6$ & $48.1 \pm 12.5$ & $48.2 \pm 13.2$ & 0.965 \\
\hline C-reactive protein (mg/L) & $0.6 \pm 0.6$ & $0.6 \pm 0.6$ & $0.7 \pm 0.7$ & 0.347 \\
\hline$\gamma$-Glutamyltransferase (U/L) & $24.3 \pm 15.9$ & $24.8 \pm 16.2$ & $21.1 \pm 13.7$ & 0.045 \\
\hline Uric acid (mg/dL) & $5.2 \pm 1.4$ & $5.2 \pm 1.4$ & $4.9 \pm 1.4$ & 0.070 \\
\hline Hypertension & 12.3 & 12.5 & 10.6 & 0.614 \\
\hline Diabetes & 4.2 & 4.5 & 2.4 & 0.360 \\
\hline
\end{tabular}

Values are presented as mean \pm standard deviation or $\%$.

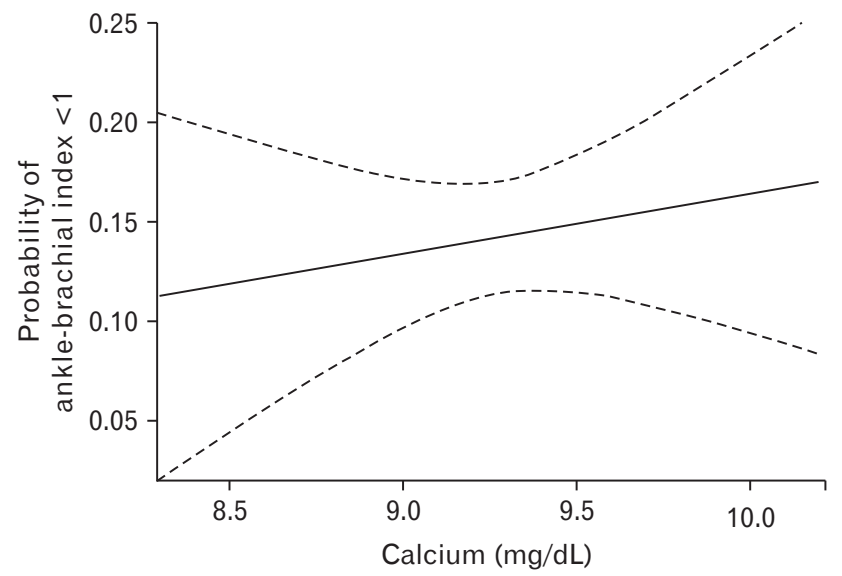

Figure 1. Unadjusted spline curve between serum calcium levels and preclinical peripheral arterial disease.

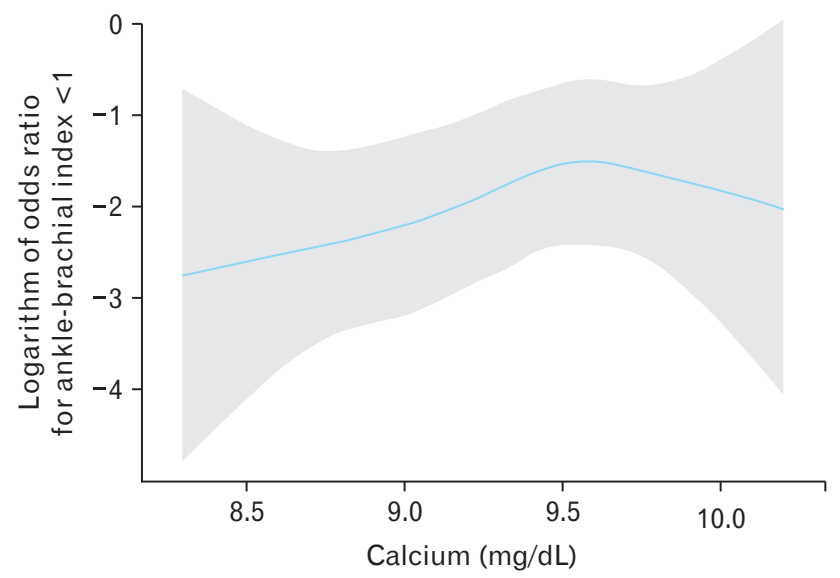

Figure 2. Adjusted spline curve between serum calcium levels and preclinical peripheral arterial disease. 
Table 2. Odds ratios and 95\% confidence intervals for preclinical peripheral arterial disease

\begin{tabular}{llc}
\hline \multicolumn{1}{c}{ Variable } & $\begin{array}{c}\text { Odds ratio }(95 \% \\
\text { confidence interval) }\end{array}$ & P-value* \\
\hline Age $(\mathrm{y})$ & $0.97(0.94-0.99)$ & 0.006 \\
Gender (female or male) & $0.92(0.42-2.01)$ & 0.837 \\
Body mass index (kg/m²) & $1.05(0.95-1.17)$ & 0.330 \\
Current smoking (yes or not) & $0.49(0.20-1.18)$ & 0.111 \\
Alcohol consumption ( $\geq 140$ g/wk or less) & $0.94(0.52-1.71)$ & 0.839 \\
Regular exercise ( $\geq$ twice/wk or less) & $1.54(0.88-2.70)$ & 0.127 \\
Systolic blood pressure (mm Hg) & $0.99(0.97-1.01)$ & 0.195 \\
Fasting plasma glucose (mg/dL) & $1.01(1.00-1.02)$ & 0.175 \\
Triglycerides (mg/dL) & $1.00(1.00-1.00)$ & 0.412 \\
High-density lipoprotein cholesterol (mg/dL) & $0.99(0.97-1.02)$ & 0.562 \\
C-reactive protein (mg/L) & $1.29(0.97-1.71)$ & 0.081 \\
$\gamma$-Glutamyltransferase (U/L) & $0.98(0.96-1.00)$ & 0.087 \\
Uric acid (mg/dL) & $0.91(0.72-1.16)$ & 0.456 \\
Hypertension medication (yes or no) & $1.15(0.46-2.87)$ & 0.770 \\
Diabetes medication (yes or no) & $0.52(0.10-2.68)$ & 0.433 \\
Hyperlipidemia medication (yes or no) & $3.67(1.33-10.14)$ & 0.012 \\
Calcium (mg/dL) & $2.28(1.02-5.11)$ & 0.046 \\
\hline
\end{tabular}

*By multiple regression analysis.

was defined as an ABI of 0.9-1.0. An ABI in this range has also been correlated to endothelial dysfunction, which is the initial step in the pathogenesis of atherosclerosis. ${ }^{5)}$ Although the endothelium is only a single layer of simple cells, it performs numerous critical homeostatic functions ${ }^{13)}$ and is the target of vascular inflammation and thrombosis. It is also the main site of atherosclerosis and its clinical ramifications, including myocardial infarction and stroke. ${ }^{13)}$ In the presence of cardiovascular risk factors, including hypercholesterolemia, hypertension, diabetes, and smoking, the endothelium has decreased nitro oxide accessibility. Ultimately, this change damages the endothelium and its ability to vasodilate. ${ }^{5,13)}$ Such endothelial dysfunction increases the risk of major cardiovascular morbidity. ${ }^{13)}$

To prevent cardiovascular events, it is clinically imperative that the treatment of atherosclerosis be properly researched before progression to advanced atherosclerosis with gravely injured vessels. ${ }^{10,14)}$ Certain mechanisms may explain the significant relationship between serum calcium levels and cardiovascular diseases. ${ }^{3)}$ Accumulating evidence demonstrates that higher serum calcium levels are associated with increased risk of cardiovascular events. For example, there is increased carotid artery plaque thickness and abdominal aortic calcification in individuals with elevated calcium levels, suggesting that calcium directly affects the vessel walls. ${ }^{3)}$ Arterial stiffness is also known to be associated with cardiovascular disease. ${ }^{15)}$ Another possible mechanism is through the binding of calcium with pyrophosphate, which is an important inhibitor of tissue calcification. In the context of excess serum calcium, there is reduced pyrophosphate, and subsequently there may be more tissue calcification., ${ }^{1,2,16)}$

PTH levels are also implicated in the relationship between serum calcium levels and cardiovascular disease. PTH inhibits arteriosclerotic Wnt/ $\beta$-catenin signaling in vascular smooth muscle cells. However, elevated serum calcium levels reduce the PTH level and thereby influence vascular wall function. ${ }^{3,17)}$

Prior studies have demonstrated a noteworthy and straightforward relationship between serum calcium level and lipids. In addition, estrogens are thought to neutralize the ominous impact of serum calcium on lipid metabolism. Likewise, serum calcium supplementation may contribute to increased serum cholesterol by diminishing hepatic catabolism in cases of estrogen deficiency. Ordinarily, estrogen activates the low-density lipoprotein cholesterol receptor and therefore enhances both cholesterol catabolism and lipid synthesis. In contrast, calcium decreases cholesterol catabolism and stimulates lipid synthesis. Hence, a combination of calcium supplementation and an absence of estrogens may impair the lipid profile and increase cardiovascular risk. ${ }^{2)}$

Our study has several limitations. First, the research population may not adequately represent the general Korean population given that the participants were otherwise healthy volunteers. We found that age is not related to the diagnosis of preclinical PAD. Moreover, since we conducted our research on healthy adults, unknown genetic factors could have influenced our results. Second, this study is a cross-sectional study, suggesting that caution should be used in causal and temporal interpretations. Additional studies in extensive, prospective investigations over an extended period of time are warranted. Third, PTH and vitamin D were not measured at the beginning of this study. Additional prospective studies in a large patient population are needed to substantiate these findings. Lastly, in the present study, as the serum calcium increases the probability of $\mathrm{ABI}$ lower than 1.0 tended to be stabilized in the adjusted spline curve. Relatively small numbers of participants with serum calcium level $>9.5 \mathrm{mg} / \mathrm{dL}$ were included in the study population.

In conclusion, our findings demonstrate that increased serum calcium levels are independently and positively associated with preclinical PAD irrespective of classical cardiovascular risk factors in apparently healthy Korean adults.

\section{CONFLICT OF INTEREST}

No potential conflict of interest relevant to this article was reported.

\section{REFERENCES}

1. Bianchi K, Rimessi A, Prandini A, Szabadkai G, Rizzuto R. Calcium and mitochondria: mechanisms and functions of a troubled relationship. Biochim Biophys Acta 2004;1742:119-31.

2. Gallo L, Faniello MC, Canino G, Tripolino C, Gnasso A, Cuda G, et al. Serum calcium increase correlates with worsening of lipid profile: an observational study on a large cohort from South Italy. Medicine (Baltimore) 2016;95:e2774.

3. Reid IR, Bolland MJ, Avenell A, Grey A. Cardiovascular effects of calcium supplementation. Osteoporos Int 2011;22:1649-58.

4. WOCN Clinical Practice Wound Subcommittee. Ankle Brachial Index: quick reference guide for clinicians. J Wound Ostomy Continence 
Nurs 2012;39(2 Suppl):S21-9.

5. Kajikawa M, Maruhashi T, Iwamoto Y, Iwamoto A, Matsumoto T, Hidaka T, et al. Borderline ankle-brachial index value of 0.91-0.99 is associated with endothelial dysfunction. Circ J 2014;78:1740-5.

6. Resnick HE, Lindsay RS, McDermott MM, Devereux RB, Jones KL, Fabsitz RR, et al. Relationship of high and low ankle brachial index to all-cause and cardiovascular disease mortality: the Strong Heart Study. Circulation 2004;109:733-9.

7. Criqui MH, McClelland RL, McDermott MM, Allison MA, Blumenthal RS, Aboyans V, et al. The ankle-brachial index and incident cardiovascular events in the MESA (Multi-Ethnic Study of Atherosclerosis). J Am Coll Cardiol 2010;56:1506-12.

8. Hsu PC, Lee WH, Chu CY, Lin TH, Su HM, Lee CS, et al. Heart rate significantly influences the relationship between atrial fibrillation and ankle-brachial index. J Cardiol 2015;66:143-7.

9. Allison MA, Laughlin GA, Barrett-Connor E, Langer R. Association between the ankle-brachial index and future coronary calcium (the Rancho Bernardo study). Am J Cardiol 2006;97:181-6.

10. McDermott MM, Liu K, Criqui MH, Ruth K, Goff D, Saad MF, et al. Ankle-brachial index and subclinical cardiac and carotid disease: the multi-ethnic study of atherosclerosis. Am J Epidemiol 2005;162:33-41.

11. Allison MA, Hiatt WR, Hirsch AT, Coll JR, Criqui MH. A high anklebrachial index is associated with increased cardiovascular disease morbidity and lower quality of life. J Am Coll Cardiol 2008;51:1292-8.

12. Aboyans V, Criqui MH, Abraham P, Allison MA, Creager MA, Diehm C, et al. Measurement and interpretation of the ankle-brachial index: a scientific statement from the American Heart Association. Circulation 2012;126:2890-909.

13. Syvanen K, Korhonen P, Partanen A, Aarnio P. Endothelial function in a cardiovascular risk population with borderline ankle-brachial index. Vasc Health Risk Manag 2011;7:97-101.

14. Iida M, Yamamoto M, Ishiguro Y, Yamazaki M, Ueda N, Honjo H, et al. Association of left atrial phasic volumes with systemic arterial stiffness and ankle-brachial index in hypertensive patients. J Hum Hypertens 2017;31:270-7.

15. Lee SH, Choi SH, Kim EK, Yang JH, Song YB, Hahn JY, et al. Borderline ankle-brachial index is associated with poor short-term clinical outcome after coronary artery intervention. Atherosclerosis 2016;249:18690.

16. Peacock M. Calcium metabolism in health and disease. Clin J Am Soc Nephrol 2010;5(Suppl 1):S23-S30.

17. Deng XR, Zhang YF, Wang TG, Xu BH, Sun JC, Zhao LB, et al. Serum calcium level is associated with brachial-ankle pulse wave velocity in middle-aged and elderly Chinese. Biomed Environ Sci 2014;27:594600 . 